\title{
EL MODELO DISCURSIVO EN LA ESCRITURA A TRAVÉS DEL CURRÍCULO
}

\section{The discursive model in writing across the curriculum}

- Nour Adoumieh

Instituto Superior de Formación Docente Salomé

Ureña, República Dominicana

\nouradoumieh@gmail.com

iD https://orcid.org/0000-0002-9784-2073

\section{Resumen}

La educación universitaria exige que el estudiante tenga una interacción constante con el discurso escrito. Dicha práctica requiere de un conocimiento que va más allá del código. Existen convenciones lingüísticas y sociales en cada comunidad discursiva, por lo tanto, los discentes requieren recibir formación y reflexionar sobre el proceso de escritura desde modelos pedagógicos. El propósito de este artículo es ofrecer la construcción teórica del modelo discursivo en la escritura a través del currículo, a fin de fomentar la función epistémica en el marco de las disciplinas, dado que la escritura ocupa un protagonismo determinante en todo contexto educativo, tanto para producir conocimiento como para demostrarlo. Algunas de las bases teóricas que sostienen este estudio son Morin (1996), Apple (2000), Van Dijk (2003), Álvarez Angulo y Ramírez Bravo (2006), entre otros. En este sentido, se realiza un abordaje teórico desde las distintas acepciones de modelo y de discurso, con el propósito de delimitar la naturaleza del constructo desarrollado. Posteriormente, se presenta el modelo con su descripción y, por último, los elementos que lo caracterizan: proceso de construcción discursiva, periferia de la competencia organizacional retórica, procesos cognitivos y acción educativa.

Palabras clave: modelo discursivo, modelo, discurso, proceso de escritura, escritura.

\begin{abstract}
The university education requires that the student interacts with the written speech. These practices require knowledge that goes beyond the code. There are linguistic and social conventions in each discursive community, therefore the students need to receive training and reflect on the scriptural process from pedagogical models. The purpose of this article is to offer the theoretical construction of the discursive model in writing through the curriculum in order to promote the epistemic function within the disciplines, given that writing occupies a decisive role in every educational context, both to produce knowledge as well as prove it. Some of the theoretical bases that support this study are Morin (1996), Apple (2000), Van Dijk (2003), Álvarez Angulo and Ramírez Bravo (2006), among others. That being the case, a theoretical approach is made from the different meanings of the model and discourse in order to delimit the nature of the developed construct. Then, the model is presented with its description and finally, the elements
\end{abstract}

ISSN (impreso): 2636-2139

Recibido: 22 octubre 2018

ISSN (en línea): 2636-2147

Aprobado: 23 noviembre 2018

Sitio web: https://revistas.isfodosu.edu.do/recie

\section{COMO CITAR:}

Adoumieh, N. (2018). El modelo discursivo en la literatura a través del currículo. Revista Caribeña de Investigación Educativa (RECIE), 2(2), 77-93. https://doi.org/10.32541/recie.2018.v2i2.pp77-93 
that characterize it: discursive construction process, a periphery of rhetorical organizational competence, cognitive processes, and educational action.

Keywords: discursive model, model, speech, writing proccess, writing.

\section{Introducción}

En este estudio abordamos un recurrente tema de interés académico, la escritura. Este proceso complejo de alto compromiso cognitivo requiere de la participación activa del escritor. Partir de la premisa de que los primeros niveles educativos capacitan a los estudiantes para comunicarse adecuadamente en el nivel universitario es una utopía, ya que se necesita de una alfabetización académica propia del ámbito universitario y de la comunidad discursiva en la que se desarrollen los estudios. Aunado a ello, con frecuencia se presentan problemas en la consolidación de esta habilidad productiva, porque se concibe desde la perspectiva de producto y no de proceso. El objetivo de este artículo es construir los fundamentos de un modelo discursivo para una hermenéutica de acción educativa, que permita la transformación de la escritura dentro de las disciplinas. Para ello, se hace un recorrido por las distintas acepciones de modelo y discurso, luego se presenta el modelo discursivo y los cuatro pilares fundamentales: proceso de construcción discursiva, periferia de la organización retórica, procesos cognitivos y acción educativa. Desde la perspectiva de este modelo, la escritura se inserta en una disciplina específica y se vale de la autorregulación de los procesos cognitivos para forjar una base que induzca a la reflexión y al análisis del género discursivo, privilegiando el carácter social y cultural de la lengua. Una comunidad discursiva se apoya en los géneros disciplinares y, a su vez, estos últimos son los que proveen el conocimiento en una disciplina. De allí, la importancia de basar el abordaje pedagógico de la escritura en el reconocimiento de los géneros a fin de promover que el participante sea miembro activo de una comunidad discursiva.

Los procesos curriculares exigen un modelo discursivo que provea de estrategias para el desarrollo de una competencia retórica que permite procesar significantes, descomponer significados y sentidos, así como identificar propósitos enunciativos. Además, se requiere propiciar el desarrollo de una actitud positiva y crítica en el individuo hacia el proceso de escritura. Todo ello exige un contexto no tradicionalista, sino crítico - constructivo, donde se privilegie el respeto colectivo hacia las ideas de los demás, con un docente mediador y pluralista que genere impacto social y promueva la transformación social y cultural.

En lo relacionado con la escritura a lo largo del currículo, cabe manifestar que los estudiantes deben adentrarse en la cultura de la escritura académica, y los responsables de esta inclusión son los profesores. Para ello, se tiene que comenzar con la capacitación de los mismos formadores de las distintas disciplinas, para que sean ellos quienes generen situaciones de aprendizaje, ya que la alfabetización académica no es un proceso acabado que se logra de una vez y para siempre. Además, a través del trabajo interdisciplinario, los beneficios son múltiples para todos los actores del hecho educativo. La falsa creencia de que la responsabilidad del desarrollo de las competencias lingüísticas concierne solo a los especialistas del área debe ser abandonada, pues a decir de Carlino (2005), el compromiso es de todos. En este sentido, la proyección de este modelo discursivo es pertinente para un programa de alfabetización académica, cuyos lineamientos y resultados serán expuestos en otro artículo. 


\section{Acepciones de modelo y de discursivo}

Desde la antigüedad, el ser humano se ha servido de determinados patrones o de lineamientos para crear representaciones de una realidad. Incluso, el método socrático, el cual ofrece a través de la dialéctica reflexiva o demostración lógica la generación de nuevas ideas podría ser concebido como modelo. Los modelos hacen una representación concreta de algo abstracto o no, para la sistematización de un fenómeno o de un proceso.

En las distintas esferas de la ciencia, se ha hecho uso de los modelos para presentar una realidad física o social, sistematizarla y comprenderla. Desde el punto de vista epistemológico, la noción de modelo, como representación, ha sido empleada con el propósito de explicar la realidad y de convertirla en reproducible. Generalmente, un modelo fomenta el desarrollo de un conocimiento flexible y crítico que puede ser aplicado de diferentes formas, pues privilegia una visión amplia de cómo la ciencia se construye y se materializa (Justi, 2006).

En los modelos prevalece siempre una teoría que le proporciona el sustento y una acción que se materializa con una realidad verificable. Cabe destacar que el modelo es abstracto, pero su operatividad pertenece al plano de lo concreto, al mismo tiempo que se integran los elementos del conjunto que lo conforman. En este orden de ideas, "los modelos parecen ser sucesivas aproximaciones a los fenómenos, construcciones que se van mejorando o adaptando a partir de la respuesta objetiva que ofrece la realidad" (Calvo, 2006, p. 21). Lo anterior permite dilucidar que un modelo determinado no es único ni excluyente, sino que es solo una manera de ver la realidad con base en determinados principios. El concepto de modelo que se aborda en este estudio no es propiamente el científico, ya que no pretende describir o explicar un fenómeno únicamente. El modelo científico se refiere a una representación circunstancial e idealizada de un fenómeno físico con la finalidad de describirlo, explicarlo y/o predecir algún proceso o fenómeno (Bunge, 1985).

En el campo de la enseńanza y el aprendizaje, la noción de modelo se dirige hacia una representación sobre determinados aspectos de una realidad con unas condiciones específicas, para conseguir un fin. Existen diversos tipos de modelos dentro de este campo, en este caso se circunscriben a los modelos didácticos y pedagógicos. Un modelo se identifica con una especie de esquema que selecciona datos de la realidad, los estructura, decidiendo qué aspectos son importantes para conocer la realidad a la que hace referencia. Los modelos de enseñanza son una especie de proyectos para enseñar que se convierten en herramientas para ayudar a los docentes a enseñar eficazmente, haciéndolo de forma más sistemática y efectiva, por supuesto alejándose de lo dogmático y lo instruccional.

En este orden de ideas, un modelo pedagógico viene siendo una construcción teórica y metodológica del proceso de enseñanza y del aprendizaje, distinto al caso de los modelos didácticos, pues estos últimos centran el interés en la enseñanza únicamente. Los modelos didácticos son estrategias prescriptivas diseńadas para cumplir metas de enseñanza específicas. Al respecto, Eggen y Kauchak (2001) plantean que la terminología prescriptiva se les atribuye porque "las responsabilidades del docente durante las etapas de planificación, implementación y evaluación de la enseñanza están claramente definidas" (p. 24).

Es por todo lo anterior que se toma la noción de modelo para abordar la práctica pedagógica de la producción discursiva escrita. Desde esta perspectiva, Álvarez Angulo y Ramírez Bravo (2006) explican que un modelo es "un constructo teórico que intenta representar la naturaleza y el trabajo de algunos objetos dominantes; busca unificar los dominios relevantes y es un soporte de la relación 
entre disciplinas; para el caso, producir textos en situaciones académicas" (p. 30). Un modelo aplicado a la producción es válido cuando es capaz de acoplarse a la realidad y adaptarse a esta, aunque no siempre sea de manera exacta.

Álvarez Angulo (2005) destaca las funciones que poseen los modelos de producción de textos, entre las cuales se presentan las dirigidas a analizar la complejidad del proceso de la actividad escrita y atender a las posibles dificultades que se pueden presentar en el momento de redactar. Otras funciones radican en analizar las dimensiones de la práctica de la enseñanza desde los ámbitos de la planificación y la revisión; diseñar instrumentos para guiar el proceso de redacción, los cuales pueden ser fichas de apoyo, listas de criterios para guiar el proceso; y regular las actividades de escritura en los contextos académicos.

Aun cuando los modelos de producción no tengan una naturaleza didáctica puramente, sino que más bien su intención es explicar el proceso de producción de textos, no es menos cierto que su fin último se fundamenta sobre la base de una pedagogía. Esta es la razón por la cual se ubica el modelo discursivo inserto en los modelos pedagógicos. Su intención es abarcar tanto la enseńanza como el aprendizaje de la habilidad escrita en un contexto social específico. Para este estudio, un modelo es un constructo teórico y metodológico dinámico y flexible, con fundamentos mediadores para promover la producción escrita en situaciones académicas de distintas disciplinas, valorando la investigación bajo una actitud crítica y reflexiva.

Desde esta panorámica se deja claro el porqué del diseño de un modelo de escritura. Ahora bien, el modelo expuesto es discursivo. En primera instancia, el discurso tiene una multiplicidad de acepciones. Van Dijk (2003) define el discurso como acontecimiento comunicativo. Se apoya en la teoría sociocognitiva y comprende la lingüística en su sentido estructural y funcional, aunado a que destaca el papel de la teoría de las representaciones sociales.

Jäguer (2003) explica que discurso es "el fluir del conocimiento y todo el conocimiento societal acumulado a lo largo de la historia. Fluir que determina los hechos individuales o colectivos, así como la acción formativa que moldea la sociedad, y que de este modo ejerce el poder" (p. 63). En este sentido, es una unidad reguladora y creadora de conciencia. Otro investigador sobre discurso es Fairclough (2003), quien lo define como una manera de crear significados sobre un ámbito específico de las prácticas sociales desde una perspectiva particular, es decir, depende del conocimiento de mundo del sujeto creador.

Link (1983), por su parte, expresa que discurso "es un concepto del habla que se encuentra institucionalmente consolidado en la medida en que determine y consolide la acción y, de este modo sirva ya para ejercer el poder" (p.63). No obstante, el discurso no debe ser considerado un concepto exclusivo del habla, sino como parte del uso de la lengua en una unidad formal del sistema lingüístico.

Todas las definiciones anteriores pertenecen al campo del análisis del discurso. Sin embargo, este modelo no trata lo discursivo aplicado al análisis del discurso o al análisis crítico del discurso, más bien está enfocado en lo contextual, es decir, en el discurso académico o disciplinar, por consiguiente, es de índole pedagógico.

El hecho de no ser un modelo de análisis no lo aleja de planteamientos como los de Foucault (1980), entre los que se destaca que, las formas discursivas están vinculadas a las relaciones de poder y conocimiento, que el lenguaje no es neutral y que a través del discurso se muestra la ideología y las acciones de poder. Tampoco se aleja de los planteamientos hallidayanos que exhiben la interrelación 
entre lo textual por su carácter sintáctico de coherencia y cohesión general, lo interpersonal con las relaciones sociales e identidades en un discurso; y lo ideacional como sistema de conocimiento y creencias del autor (Halliday, 1994).

Para este estudio, se asume como discurso una situación o un acto comunicativo oral o escrito, inserto en un contexto social específico, con una marcada intención comunicativa y pragmática para construir y/o transmitir conocimiento. El discurso es un acto generador de conocimiento y promotor de un pensamiento crítico y reflexivo. El trabajo pedagógico en la producción escrita contempla el discurso como una unidad de sentido que produce significados a través de la generación de conocimientos. Se inserta en un contexto comunicativo con un escenario real y participantes reales, es decir, acoplado a determinados convenios sociales, culturales e incluso temporales establecidos por una comunidad disciplinar.

Por consiguiente, asumir lo discursivo en el modelo implica involucrar en el proceso de escritura la organización retórica del texto trabajado con los propósitos comunicativos, y a su vez, circunscrito al género discursivo. Lo discursivo apunta hacia una orientación social que convierte procesos cognitivos en interacción verbal, para formar parte de un acto que busca que los interlocutores entiendan los enunciados, en otros términos, implica unas acciones cooperativas que se hacen con el fin de ser comprendidas por el otro. La organización de las acciones sociales se condiciona directamente a través del discurso.

Si se considera que todo discurso depende de una situación externa que lo rodea, y que responde a propósitos comunicativos; es imprescindible dejar a un lado el contexto disciplinar en las prácticas sistematizadas de escritura.

\section{Modelo discursivo}

El modelo discursivo es un modelo de producción de textos escritos basado en la complejidad de la construcción discursiva (Adoumieh, 2015). Este, privilegia los convenios lingüísticos establecidos en una disciplina específica. A través de la autorregulación de los procesos cognitivos, crea un marco de condiciones para reflexionar y analizar el género discursivo y luego, haciendo uso de una competencia organizacional retórica, el escritor produce un texto de manera recursiva. Este modelo privilegia el carácter social y cultural de la lengua. Lo complejo, sociocultural y dialéctico se tornan elementos clave para representar la realidad y crear una acción transformadora. Este modelo se enfatiza en la perspectiva teleológica.

El ambiente de trabajo se basa en un aprendizaje autónomo, colaborativo y asistido por tutorías. Por considerar que la escritura es una actividad humana compleja, la producción escrita es externa, pues las condiciones de cognición y comunicación requieren de un ambiente distinto al salón de clases. Esto quiere decir que el trabajo colaborativo dentro del salón se dirige hacia el análisis y reflexión sobre lo ya escrito, el tipo de texto y la dialogicidad discursiva, atendiendo a los roles discursivos. La retroinformación entre participantes promueve el aprendizaje, tanto en quien la recibe como en quien la suministra. De este modo, el diálogo permite crear conciencia sobre las prácticas discursivas inherentes a un ámbito científico en particular, al tiempo que refleja los modos de organización del discurso de esa esfera social y cultural.

Este replanteamiento conduce a un cambio educativo en el que el contexto juega un papel predominante por actuar como puente para establecer relaciones entre estructuras lingüísticas y estruc- 
turas sociales-culturales. Es el contexto del texto, acompañado de su contexto, lo que genera una representación simbólica acorde a la disciplina en la que se escribe, pues la escritura es una actividad socialmente cooperativa y se fortalece entre los pares. La escritura a través del currículo supone una construcción del discurso mediante estructuras prototípicas, léxicas, relaciones sociales, formas de razonamiento propias de una esfera social.

En correspondencia con lo anterior, el modelo posee elementos importantes que se deben explicar para entender su funcionamiento. Principalmente, se destaca que este modelo, apoyado en la pedagogía crítica, no responde a un método prescriptivo, sino más bien dinámico. Se caracteriza por concebir la escritura como proceso social adjunto a una comunidad académica. Algunas investigaciones como las de Ferreiro (1994), Tolchinsky (1993), Tolchinsky y Simó (2001), e incluso modelos muy reconocidos como los de Bereiter y Scardamalia (1992), o Flower y Hayes (1981); se centraron en los procesos cognitivos orientados a la producción escrita de textos. Sus aportes a la enseńanza de la lengua han sido positivos y trascendentales; sin embargo, aspectos dirigidos hacia la interacción social, la competencia organizacional retórica y la construcción y negociación de significados y sentidos no han sido considerados.

Las estrategias metalingüísticas son importantes dentro de los procesos de escritura, pero son las acciones pedagógicas las que deben fortalecer los procesos cognitivos de reflexión, análisis y construcción retórica que le permitan al escritor reconstruir el conocimiento en función de propósitos comunicativos, acordes a la comunidad discursiva en la que escribe. Estos procesos activan actitudes dirigidas hacia la toma de conciencia crítica sobre la organización del conocimiento que el escritor pueda tener frente a una intención de escritura con fines específicos. Para explicar detalladamente la articulación de todos los elementos que caracterizan el modelo, se presenta la siguiente estructura: proceso de construcción discursiva, periferia de la competencia organizacional retórica, procesos cognitivos y acción educativa (ver figura 1).

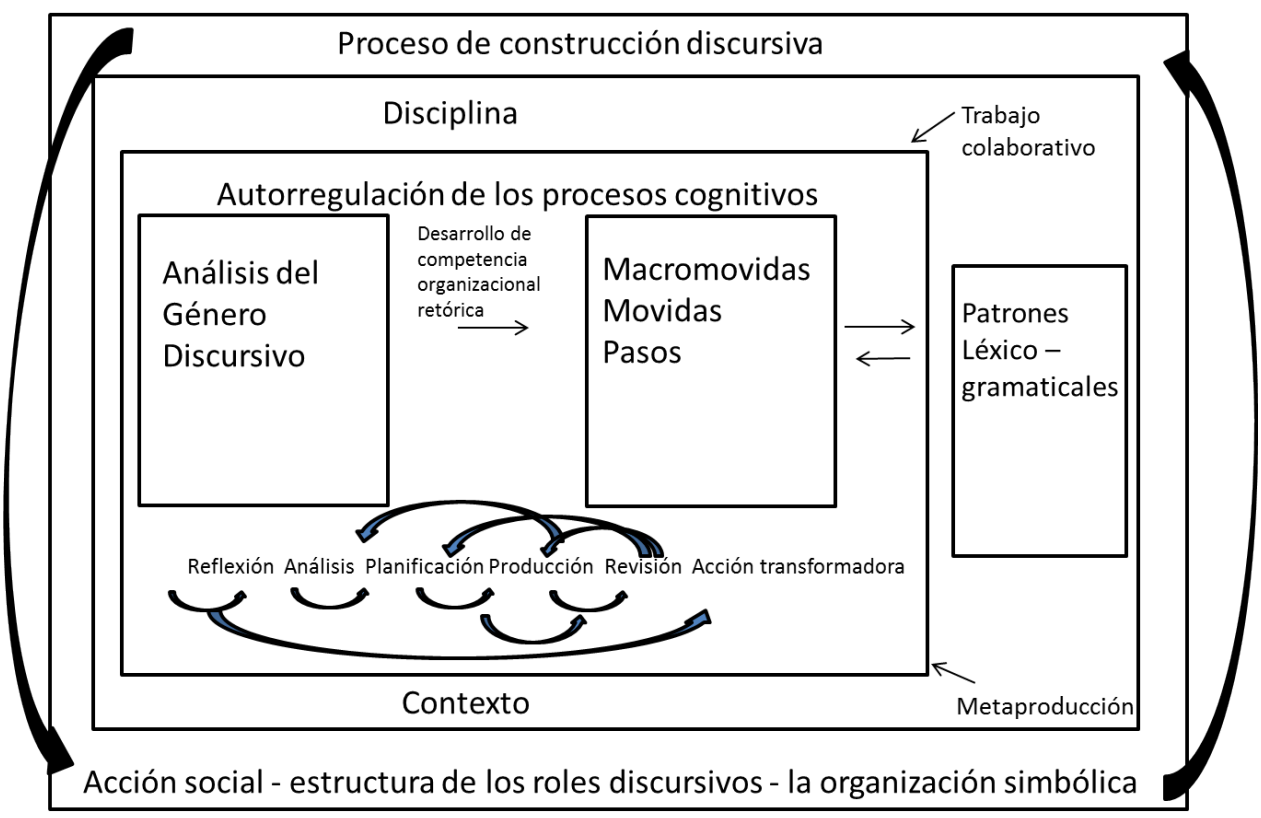

Figura 1. Esquema del modelo discursivo. Fuente: Adoumieh (2015). 


\subsection{Proceso de construcción discursiva}

Para el modelo discursivo, el proceso de construcción discursiva escrita se basa en un problema retórico dirigido hacia las necesidades sociales específicas de la comunidad donde se escribe, es decir, en el ámbito universitario debe enfocarse en temas relacionados al saber disciplinar. El propósito de la producción escrita estriba en la generación de conocimiento que permita una transformación social en el sujeto e incluso en su audiencia. Bajo una visión somera y simplista, la escritura podría basarse en transmitir información únicamente. No obstante, este modelo, circunscrito al paradigma de la complejidad, busca, a través del cuestionamiento y del desarrollo de la competencia organizacional retórica, que el escritor entienda el porqué de lo que está escribiendo, y que, de esta manera, pueda hacer uso de la escritura en su nivel epistémico y no solo instrumental.

Cada actividad de composición es un acto contextualizado que dependerá de un tiempo y espacio específico con interlocutores concretos que comparten un mismo código. Además, locutores e interlocutores deben compartir una concepción de mundo, unos conocimientos enciclopédicos, una misma base cultural. Esa contextualización permitirá economizar todo lo posible (Principio de cooperación de Grice, 1975).

Para ello se presenta el cuestionamiento como un mecanismo de autorregulación del conocimiento propio a través de seis preguntas que presentamos como el hexágono de la complejidad de la construcción discursiva (ver figura 2). Aquí la organización del conocimiento se hace alrededor de un problema retórico.

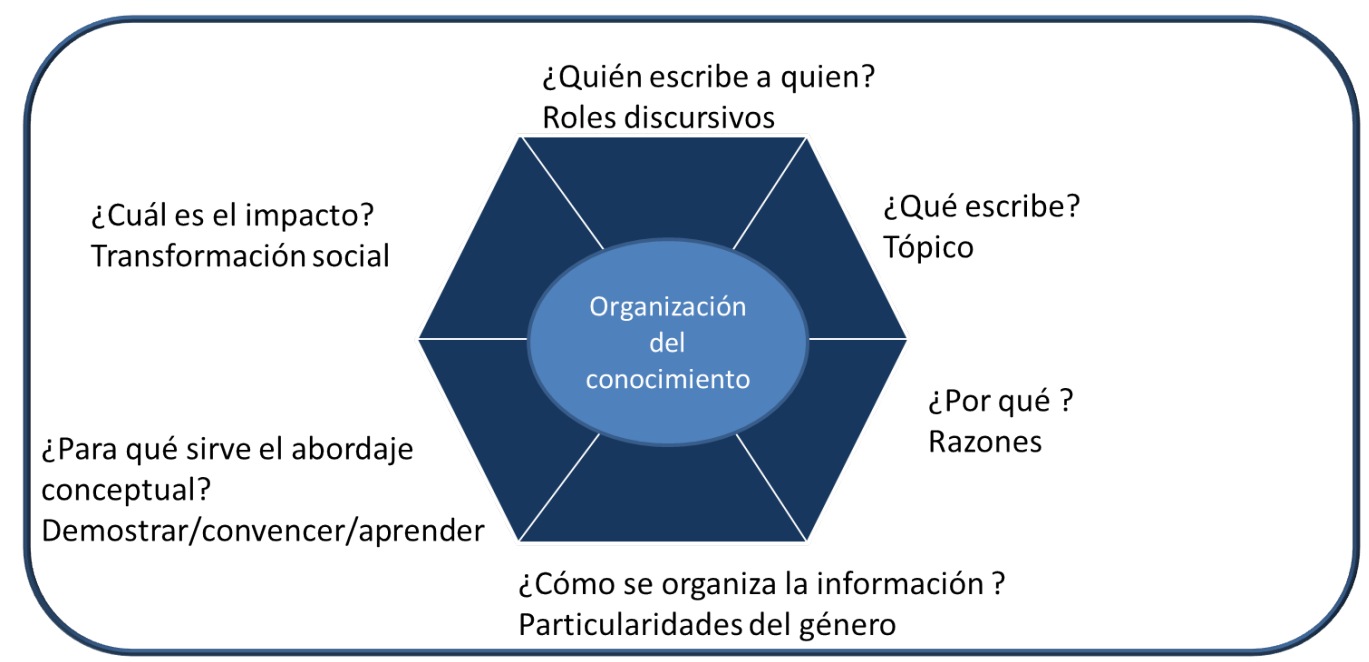

Figura 2. Hexágono de la complejidad de la construcción discursiva: Organización del conocimiento alrededor del problema retórico. Fuente: Adoumieh (2015).

El centro del hexágono es formado por la organización del conocimiento, la cual requiere de una planificación previa y de la activación de procesos cognitivos, y a su alrededor se configuran cuestionamientos retóricos. En cuanto a quién escribe a quien y los roles discursivos, se refiere a las personas que se interrelacionan y regulan el flujo discurso. En la escritura académica, el locutor-escritor es el estudiante que en la mayoría de los casos se dirige a sus docentes o a pares académicos; sin embargo, podría ser también a miembros expertos de la comunidad discursiva. 
Existen también otras voces que se evocan por el locutor, directa e indirectamente a través del discurso referido. Para la escritura a través del currículo, es sumamente necesario el uso de la polifonía textual, porque la generación del conocimiento no parte de la nada, sino de conocimiento que se torna como compartido. Se introduce otro enunciador en el propio texto para respaldar lo dicho, darle credibilidad o para presentar objeciones. Los textos producidos nunca pueden ser singulares, ya que están cargados de textos elaborados por diferentes hablantes en los mismos contextos y con las mismas intenciones.

La heterogeneidad se da porque los textos responden a una esfera determinada que hace que se tengan deudas con nuestros antecesores. Todo lo que se dice, de algún modo, es polifónico o intertextual, ya que incluye muchas voces y conecta con ideas de otros textos. Reyes (1995) afirma que somos prisioneros de una lengua que heredamos cargada de voces, connotaciones, puntos de vista que estamos obligados de repetir. En el caso del qué escribe, debe ir integrado a la disciplina y lo que culturalmente se espera en ese círculo social. Además, se adhiere a un objeto o problema real que se presenta como pretexto para generar conocimiento en el contexto disciplinar. En lo concerniente al por qué, se refiere a las razones epistemológicas y pragmáticas de la disciplina específica, las cuales impulsan el desarrollo de actividades particulares. No todas las producciones escritas de un ámbito académico tienen las mismas estructuras, ni los mismos motivos. Aunque todos transmiten información, son diferentes y responden a distintos objetivos. Los efectos que pretenden conseguir en el lector también son variados.

Lo antes expuesto debe girar en torno a una manera de presentar el conocimiento, y esto es lo que se hace a partir de las particularidades de cada género discursivo y responde a la pregunta ¿Cómo se organiza la información? Cada género discursivo tiene sus particularidades atendiendo a la definición bajtiniana, en la que los géneros se relacionan con las prácticas sociales humanas y dependen del contrato social establecido por una esfera social específica (Bajtín, 1979). Desde esta visión, el lenguaje es visto como práctica social que se integra con otras prácticas humanas. Se emplea con distintos fines según las convenciones de la esfera social y los enunciados adquieren el sentido según las circunstancias. La inclusión del análisis de macromovidas, movidas y pasos tiene fines eminentemente didácticos que pudieran facilitar la apropiación del género, pues le permite al participante reflexionar sobre la estructura retórica de los segmentos discursivos que se han tomado como textos modélicos, es decir, el mediador selecciona algunos modelos prototípicos para ser estudiados y así hallar en ellos los propósitos comunicativos.

Todo lo anterior debe dejar claro para qué sirve el abordaje conceptual. El estudiante debe demostrar que se conoce cabalmente la temática, convencer y fundamentar las ideas sobre un tema y aprender de lo que se está diciendo. El fin último de la escritura con carácter social implica concienciar a los miembros de la comunidad discursiva sobre la responsabilidad que tiene el locutor con lo dicho, e incluye el fortalecimiento del pensamiento propio para asumir los problemas retóricos y las alternativas posibles para presentarlo como un conocimiento sistematizado y contribuir así con la comunidad científica.

Por otro lado, Montolío (2000) explica que la fase de la documentación es clave en el proceso de escritura, y que se inicia recabando toda la información interesante para el tema a desarrollar. Luego, de manera minuciosa y a través de un complejo trabajo intelectual, se establece la relación presente entre la información recabada. Finalmente, la valoración crítica es el paso más importante para el logro de una integración coherente y que, a su vez, represente un conocimiento organizado. 
$\mathrm{Al}$ presentar tridimensionalmente el hexágono (ver figura 3), se nota que el ápice es el problema retórico y que alrededor de este se presentan los cuestionamientos para organizar el conocimiento y hacer la construcción discursiva desde una óptica eminentemente socio-contextual.

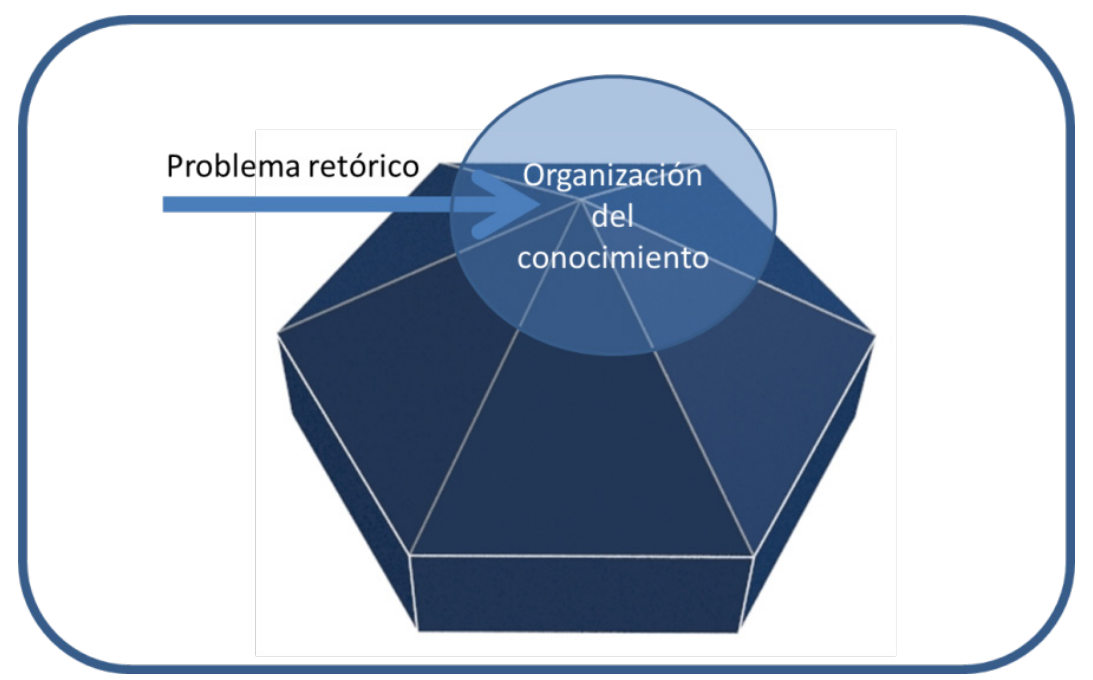

Figura 3. Presentación tridimensional del hexágono de la complejidad discursiva. Fuente: Adoumieh (2015).

El conocimiento se constituye a partir de experiencias previas y de modelos de pensamiento o representaciones sociales que el locutor recibe y, al mismo tiempo, transmite a través del uso y la práctica social. De este modo, este conocimiento es, en muchos aspectos, socialmente elaborado y compartido en la comunidad. La construcción discursiva se convierte en vehículo transmisor de puntos de vista, forma modelos sociales y crea cogniciones. Aprender a interpretar los hechos y a reconstruir las interpretaciones ajenas es una necesidad. La lengua no refleja la realidad, si hay texto es porque alguien lo dijo, y si lo dijo un sujeto, quiere decir que es subjetivo. Está modalizado según la intención del escritor. Generalmente, el escritor busca dominar el entorno y, a su vez, comprenderlo, explicarlo y situarse como locutor-escritor frente a la situación enunciativa. En otros términos, se trata de hacer uso práctico y contextualizado de los convenios preestablecidos culturalmente (ver figura 4).

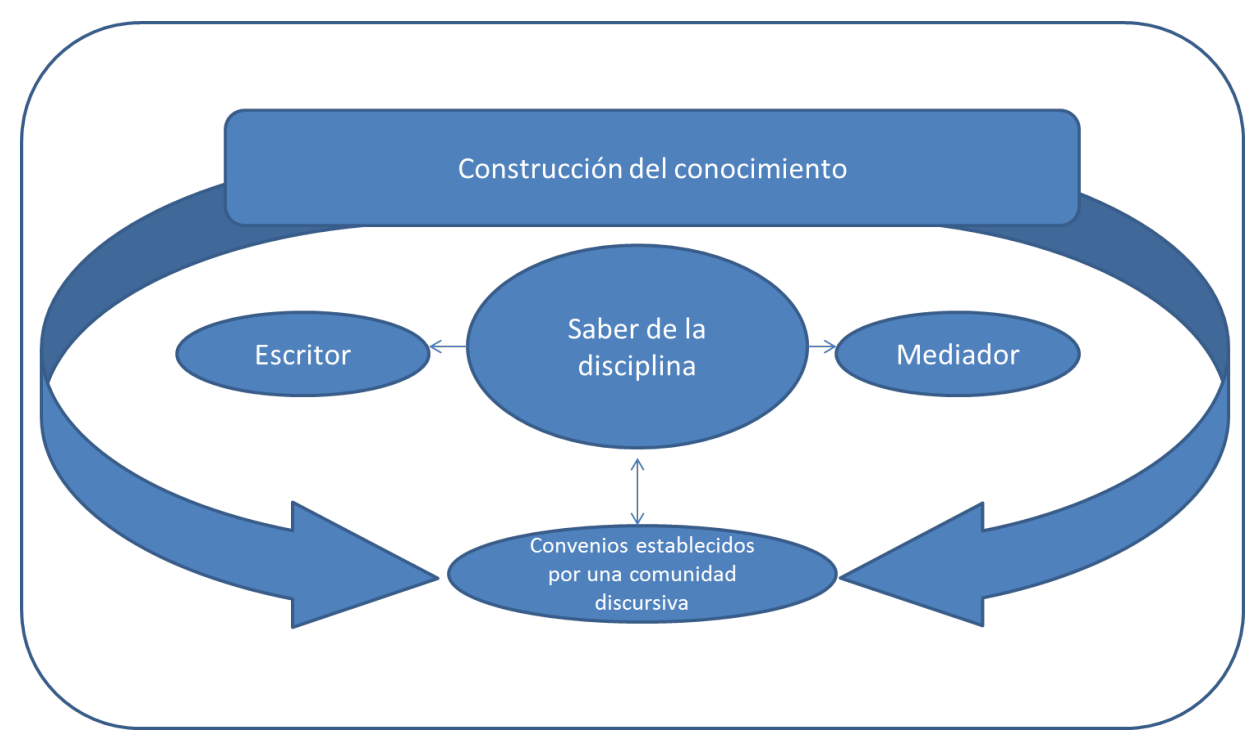

Figura 4. Operatividad de la construcción del conocimiento. Fuente: Adoumieh (2015). 
Desde esta perspectiva, la producción escrita es concebida como un proceso cognitivo socio-cultural inherente al ser humano y herramienta imprescindible en la formación académica en todos los niveles educativos. La escritura académica demanda el conocimiento de una organización retórica y exige del uso de determinados estilos lingüísticos, productos de esos acuerdos establecidos culturalmente por cada disciplina.

Para Vigotsky (1973), la escritura organiza los procesos psicológicos superiores valiéndose de la autorregulación voluntaria, la realización consciente y la mediación, lo que quiere decir que es el individuo quien debe controlar sus operaciones mentales, su posición ante lo escrito, la forma como presenta el problema. Es el escritor quien de manera consciente construye discursivamente su intención comunicativa.

El proceso de la composición escrita es dinámico y abierto, el mensaje se construye a partir de la interacción entre los conocimientos previos y el escrito, por ende, su contenido nunca será el mismo para todos los lectores. Esto se representa con el esquema de la construcción del conocimiento. El círculo principal está conformado por el saber de la disciplina y su contexto social, luego se consideran las convenciones discursivas para el logro de los propósitos establecidos, para ello, el escritor activa sus conocimientos previos conceptuales y estructurales. El mediador vela para que se construya el significado y se presente según los preceptos esperados por los miembros de la comunidad discursiva a la que se pertenece.

Para formar parte de una comunidad discursiva, por lo tanto, los sujetos que quieran ingresar deben ser aprehensivos con las normas estipuladas, con las formas discursivas apropiadas para ese círculo. De esta manera, el locutor-escritor exhibe que conoce el campo profesionalmente y que asume una postura frente al saber, es decir, muestra que su producción escrita es acorde al ámbito y que también conoce lo que otros han investigado al respecto.

\subsection{Periferia de la competencia organizacional retórica}

Para este estudio, la competencia organizacional retórica es la capacidad de interactuar discursivamente en el marco de un acto comunicativo, para producir textos en contextos situados. Los textos escritos son unidades coherentes y adecuadas a la situación y al tema. Al mismo tiempo, son sistemas complejos de unidades lingüísticas de diferentes niveles y de reglas y de criterios de organización empleados por la escritura, debido a la naturaleza pragmática del canal escrito. Esta competencia implica que el escritor debe desarrollar habilidades para comprender y construir organizacionalmente la estructura prototípica del género, respondiendo a la temática disciplinar donde ocurre el acontecimiento.

Para que este desarrollo se dé, el escritor construye una hipótesis sobre la situación comunicativa basada en interpretaciones, producto de inferencias realizadas desde el análisis del género discursivo. Esos análisis se hacen a textos modélicos, de la disciplina en la que se esté trabajando. Además, el escritor es activo, cuyo rol discursivo está vinculado a autopresentarse como miembro de una comunidad específica, razón por la cual se aborda la estructura retórica global del tipo de texto a escribir y se reconocen los patrones léxico-gramaticales propios de ese ámbito. Los contenidos abordados dentro de este modelo discursivo de producción escrita son eminentemente los que corresponden a una esfera social específica. La figura 5 muestra la dinámica establecida: 


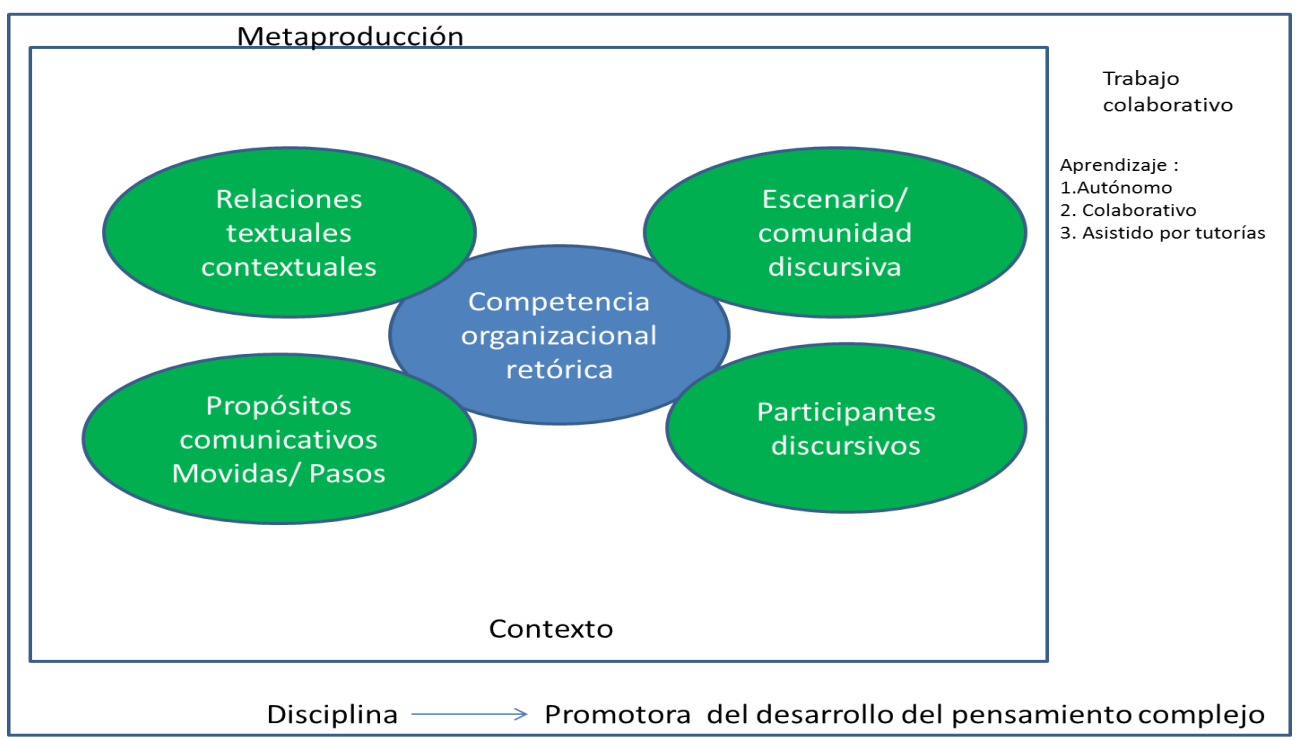

Figura 5. Periferia de la competencia organizacional retórica. Fuente: Adoumieh (2015).

La reflexión y la discusión sobre la estructura de la producción escrita hace que el escritor-locutor detecte y sea capaz de comprender el aprendizaje adecuado, tanto de su texto como de otros, dicho en otras palabras, el proceso de reflexión organizacional y la detección de rasgos o propósitos comunicativos propios de cada parte del escrito, le facilita la resolución de problemas retóricos, lingüísticos, gramaticales, pragmáticos e incluso estilísticos. Además, permite que el escritor-locutor comprenda la dinámica enunciativa dada entre los participantes discursivos, posición defendida, situación social, función o propósitos establecidos, entre otros aspectos.

Todo lo antes mencionado se presenta en el marco de una disciplina, ya que se espera hacer uso de la escritura desde el plano de lo real. Esto hace que las necesidades propias de cada grupo científico puedan ser abordadas significativamente. La disciplina es vista como:

(...) el vínculo entre ciencia y enseñanza, porque es la manera en que se institucionaliza el conocimiento: modo de dividir el saber y organizar la enseñanza. Desde que nacen, en la alta Edad Media, las corporaciones universitarias administrativamente se organizan a partir de las disciplinas que enseñan (Pedroza Flores, 2006, p.71).

Es así como la disciplina actúa como promotora del desarrollo del pensamiento complejo, en el marco de una metaproducción escrita. Para ello, recurre a un proceso de aprendizaje sobre el uso de la lengua que permita integrar los saberes. La metaproducción es acompañada del trabajo colaborativo, el cual insta a obtener un aprendizaje autónomo, colaborativo y asistido por tutorías.

Es de hacer notar que los cuatro óvalos que circundan la competencia organizacional retórica están insertos en un contexto de disciplina y que su interacción es indispensable para el desarrollo de la competencia.

\subsection{Procesos cognitivos}

Desde los inicios de los estudios psicosociolingüísticos, se ha otorgado una marcada relevancia a la necesidad de aprender a pensar como el verdadero camino para lograr el aprendizaje significativo, 
tanto de lengua como de otros conocimientos. Realmente, se pretende que la escuela fomente en los aprendices el desarrollo de los procesos cognitivos básicos, para que sean aplicados no solo en el entorno académico, sino también cotidiano (Ríos, 2004). A fin de cuentas, enseñando a aprender es que se logra el aprendizaje para la vida. Se trata de una cognición situada y contextuada que pretende llevar al sujeto cognoscente a la construcción y (re) construcción de las situaciones en beneficio del desarrollo del pensamiento lógico y crítico, capaz de hacer que el ser humano logre obtener nuevos conocimientos.

En este modelo de producción escrita académica, se articulan seis procesos cognitivos, a manera de fases, y se trabajan de forma recursiva: reflexión, análisis, planificación, producción, revisión y acción transformadora. Cada uno de estos genera discusión sobre la construcción del propio escrito, en cuyo proceso se crean nuevos problemas sobre los cuales hablar o discutir.

La reflexión es vista como proceso que puede ser desarrollado a través de prácticas guiadas. Su aplicación en la construcción discursiva constituye el centro en el que el paradigma crítico se sustenta, al mismo tiempo se basa en postulados del aprendizaje significativo y socio-cultural. El estímulo de diálogos y experiencias grupales mediadas ofrece la oportunidad de construir significados alrededor del problema retórico. La reflexión juega un papel importante en la autorregulación de lo conceptual procedimental, lo actitudinal.

El desarrollo de la producción escrita requiere de la organización de los pensamientos, reflexión en los enunciados y una capacidad crítica para reconstruir las ideas en función del otro sujeto. Escribir y comprender requieren de la activación de procesos cognitivos y a la vez son recursivos e interdependientes. Para Schön (1992), la reflexión es acción, es pensar en lo que se hace, mientras se está haciendo. Permite hacer inferencias a partir de los hechos que se observan. Al escribir, se requiere de una comprensión previa, organización de la información y sobre todo la criticidad de formular los planteamientos según las necesidades personales y de los destinatarios. Igualmente, sucede que en el procesamiento de la información, se usa lo escrito por otros y se reconstruye la intención comunicativa, activando conocimientos previos, el contexto, ¿y por qué no?, el contexto de lo leído.

En cuanto al análisis, proceso cognitivo a través del cual se descompone un todo en sus partes, se hace atendiendo a criterios de organización retórica del tipo de texto, al género discursivo, los propósitos comunicativos y las marcas gramaticales y discursivas que generen evidencia acerca de las características buscadas. La intención es establecer criterios que permitan la separación de los elementos y que también se reconozca la importancia de establecer las relaciones presentes entre ellos, a fin de que el análisis sea sustancial y productivo. Asimismo, el análisis es empleado desde la perspectiva conceptual para comprender la información y organizar el conocimiento. Este proceso se apoya en la reflexión y genera una visión integral que se articula favorablemente con la planificación.

Desde esta óptica, la planificación es una etapa paralela que no solo requiere de un conocimiento conceptual, sino también organizacional y social. La planificación es una representación abstracta en la que el escritor se formula interrogantes con respecto a los aspectos funcionales descritos, para así dar inicio a su plan de escritura, el cual se rediseña constantemente conforme se hace la investigación y se enriquece gracias a la búsqueda de información, bien sea en la memoria de largo plazo del escritor, o en fuentes externas a través de la indagación.

Ya en el proceso de producción, el escritor convierte sus ideas en un acto concreto y elaborado, atendiendo al plan de trabajo anteriormente diseńado y activando estrategias de decisión y de selección. En este espacio se plasman las ideas con el orden previsto en el plan. Lo más importante es 
entender que, en este proceso de producción, el escritor-locutor realiza operaciones que van más allá de la traducción de paquetes semánticos alojados en la memoria a largo plazo en torno a información organizacional, de contenido y comunicativa; sino que paralelamente hace cuestionamientos críticos según lo esperado por la audiencia o los interlocutores. También, vela por la adecuación, coherencia, cohesión, entre otras propiedades textuales.

Simultáneamente a todos los procesos anteriores, se encuentra la revisión, cuyo principal objetivo es mantener el equilibrio discursivo realizando los ajustes necesarios en la producción. A través de esta operación, se evidencia el carácter recursivo de toda construcción discursiva al hacer modificaciones para mejorar el texto y contrastar los objetivos planificados con la composición realizada, a fin de retocar y mejorar el escrito. La revisión externa con los miembros de una audiencia crítica es vital, e incluso, el borrador se revisa a la luz de las expectativas del interlocutor o lector potencial.

Conforme se logre que el escritor-locutor esté consciente de la importancia de aprender a aprender, que el manejo del discurso tiene relación directa y se interconecta con su vida real, podrá otorgarle el valor y la aplicación adecuada. En eso estriba el proceso de acción transformadora. De este modo, el aprendizaje institucionalizado se torna transferible a la vida misma, lo que repercute en ganancias al considerar que la adquisición progresiva de la construcción del discurso implica el desarrollo de capacidades cognitivas superiores. La estructuración del pensamiento se ve fortalecida con la escritura y viceversa. Es oportuno citar a Giroux (1990) "aprender a escribir no significaría ya aprender a desarrollar un sistema de distribución instrumental, sino como lo ha dicho el doctor Carlos Baker, aprender a pensar. La escritura es, en este caso, una epistemología, una forma de aprendizaje" (p. 105). Esta visión se ha circunscrito, generalmente, al uso de la lengua como acción en un contexto sociocultural. Por lo dicho anteriormente, la competencia organizacional retórica y el manejo crítico de la información convergen en la construcción de significados para cumplir con los propósitos según diversos contextos.

Es conveniente recalcar que este modelo de producción es de textos académicos, los cuales constituyen una elaboración intelectual que busca abordar una temática de manera profunda y sistemática circunscrita a una disciplina. Asume el abordaje discursivo como una red de relaciones mentales estructuradas según el contexto y los lectores.

\subsection{Acción Educativa}

Todo modelo de producción de textos lleva de modo implícito una proyección didáctica que contribuye a favorecer la enseńanza y el aprendizaje de la escritura. Apple (2000) establece que es el docente el encargado de ejercer la habilidad para reproducir en su acción educativa modelos pedagógicos, ideológicos, sociales y culturales que favorecen una reflexión crítica. Ello se traduce en que el empleo de determinados enfoques conduce al logro de los aprendizajes dirigido hacia los procesos, más que a un producto, y de este modo, generaría un conocimiento en quien lo utiliza, es decir, se presenta el valor epistémico de la lengua.

En el caso del modelo discursivo, centra su interés en la escritura bajo un enfoque de procesos, por lo tanto, se enfatiza en la autorregulación de los procesos cognitivos de producción. Para el logro de este fin, se vale de la reflexión, el análisis y de estrategias metalingüísticas antes, durante y después de la producción escrita. Su orientación debe guiarse hacia conocimientos temáticos, lingüísticos, procedimentales y estructurales propios de cada género discursivo, lo cual crea un andamiaje para 
abarcar y comprender las macromovidas, movidas y pasos desde la comunidad académica como un evento social. Se sugiere enseñarle al escritor-locutor que la reescritura y la revisión recursiva son aliadas y su articulación permite obtener un escrito de mayor calidad, tanto conceptual como estructural. La actitud positiva del escritor hacia el escrito y la forma de presentarlo es relevante. En consecuencia, se debe enfatizar en el desarrollo del pensamiento, en la preparación del texto respondiendo a factores externos, como por ejemplo la audiencia y los miembros de la comunidad discursiva en la que se inserta.

Esto se hace transferible a los fundamentos del pensamiento complejo (Morin, 1996), puesto que se aborda la escritura a través del currículo como fenómeno de procesos continuos de cambio de orden y de re-organización. La construcción del conocimiento conceptual y discursivo depende de la reflexión y el análisis del tejido de las relaciones entre las partes que se configuran entre sí para formar un todo.

El procedimiento metodológico para la aplicación de este modelo es viable por medio de un programa académico de escritura a través del currículo, cuyo diseño debe responder a proyectos de aprendizaje dirigidos hacia la reflexión del proceso de escritura, análisis del género discurso, planificación de la escritura, producción, revisión recursiva y acción transformadora. Dentro de esta concepción, el trabajo colaborativo es indispensable y es la mediación pedagógica la que promueve el aprendizaje autónomo, compartido y asistido por tutorías. De esta manera, se lograría el desarrollo de competencias cognitivas y también de competencias sociales promovidas por la interacción grupal.

En el aprendizaje individual, el escritor se fundamenta teóricamente y procesa la información aplicando sus estrategias metacognitivas en la actividad de aprender y autorregular el proceso, desarrollando el pensamiento crítico. El aprendizaje colaborativo o compartido es para confrontar el texto con la mirada de los otros como sujetos interlocutores, participantes de la misma comunidad discursiva, y así validar saberes construidos individualmente con sus pares académicos e incluso con el mediador. Mientras que el aprendizaje por tutorías se hace guiado por un tutor mediador para confrontar, resolver inquietudes y responder interrogantes, y así validar el saber para mejor la construcción discursiva. En los tres tipos de aprendizajes descritos (individual, colaborativo y por tutorías) se abarcan los conocimientos conceptuales, lingüísticos, procedimentales y estructurales.

En cuanto a los integrantes de la acción educativa, se encuentra el participante del programa académico como escritor-locutor, a saber, el sujeto protagonista del proceso de aprendizaje y desarrollo de competencias y de la construcción discursiva. Su rol es activo. El docente es el mediador responsable de diseñar, en conjunto con los participantes, las estrategias, así como de guiar cooperativamente el proceso de formación en función de la resolución del problema retórico presentado. Su rol estriba en propiciar la formación de un sujeto que sea capaz de transformar su sociedad y que la interacción discursiva y el diálogo permanente le permita construir conocimiento a través de la activación de los procesos mentales.

Por otro lado, los pares son los participantes del programa, quienes forman parte de la misma comunidad científica y, su función es de prestar apoyo para sugerir cambios que mejoren la calidad de los escritos. Las sugerencias de los pares contrastadas entre sí, pero es el escritor quien posee la última palabra.

Esta perspectiva, supone un cambio epistemológico o paradigmático. Stenhouse (1987) expresa que la actividad central del ambiente de aprendizaje debe ser el diálogo a diferencia de la instruc- 
ción, puesto que esto le dará a los participantes la oportunidad de aprender mientras se expresan, al tiempo que hay una negociación de saberes. El docente pasa a ser el mediador entre el contenido lingüístico y las acciones. Esto lleva a la autocorrección y la correvisión; y permite que los participantes reflexionen de manera metacognitiva sobre sus producciones en función de mejorarlas.

Ahora bien, acorde a la dinámica sostenida, la evaluación es de tipo formativa orientada hacia la valoración de los procesos de construcción discursiva mediante la generación de borradores y del conocimiento presentado. Enfrentar esta práctica implica el desarrollo de mayor conciencia y dominio de los procedimientos que se llevan a cabo cuando se escribe, y de las competencias alcanzadas. Además, es asumir que la evaluación no es un acto para juzgar los escritos realizados, sino para fortalecerlos e impulsar al escritor hacia una actitud positiva sobre la escritura. Esto genera la posibilidad de confrontar ideas, diferenciarlas, compararlas según su uso en contextos definidos y los propósitos comunicativos específicos esperados por una comunidad científica. La autoevaluación y la coevaluación son aplicadas en este tipo de proceso, y generan procesos de metaprodución y reflexión, tanto sobre lo escrito como sobre el hecho educativo.

La adopción de la evaluación formativa, como una concepción crítica que propenda a mejorar los procesos pedagógicos y a construir el conocimiento, a la vez que privilegia la significación simbólica a través del discurso escrito. Esto generaría mayor rigurosidad conceptual, dada la organización del propio discurso, comprensión de estructuras prototípicas que redundan en beneficio de adquirir nuevas formas de conocimiento, consciencia de que el modo en que se dan los procesos cognitivos involucrados repercute en el conocimiento mismo y en el desarrollo de altos niveles de coherencia conceptual. Por consiguiente, la evaluación es dinámica y su propósito es promover el aprendizaje

Se trata de hacer el abordaje pedagógico desde una perspectiva transversal que implique una visión profunda de la enseńanza, como un acto de despertar la conciencia sobre los propios procesos adoptados en la materialización de las ideas, es decir, de la abstracción a la concreción de los procesos mentales realizados para el logro de las metas dispuestas. Por tal razón, este modelo articula lo conceptual y discursivo para propiciar la reflexión y regulación del conocimiento a través de una práctica estructurada que genere impacto en la transformación intencional de los procesos, objetos de estudio y de los participantes, con el fin de que el individuo asuma acciones y mejore su calidad de vida. Al mismo tiempo, mejora los procesos de interacción crítica y reflexiva en la construcción social del conocimiento.

\section{Reflexiones finales}

El modelo reconoce que la producción escrita es una construcción discursiva que se nutre del diálogo y privilegia el proceso de reconstrucción externo al salón de clases, pues requiere de la activación de procesos cognitivos, búsqueda de información y organización metódica de las ideas a través de la recursividad como subproceso clave. De esta manera, se postula un modelo que evidencie como finalidad el desarrollo de la escritura circunscrito a una comunidad científica, y no centrado en el aprendizaje de normas y preceptos lingüísticos. Esto quiere decir que se reconoce el carácter social y cultural de la lengua. Lo complejo, sociocultural y dialéctico se tornan elementos relevantes pues se busca hacer una representación de la realidad.

La conformación de un equipo interdisciplinario capaz de reconocer el protagonismo de la escritura en las distintas disciplinas es indispensable, pues únicamente en equipo se podría lograr una 
visión integral de la escritura. Los procesos curriculares exigen un modelo discursivo que provea de estrategias para el desarrollo de una competencia retórica que permite procesar significantes, descomponer significados y sentidos e identificar propósitos enunciativos. Además, propicia el desarrollo de una actitud positiva y crítica en el individuo hacia el proceso de escritura. Todo ello exige un contexto no tradicionalista, sino crítico-constructivo, donde se privilegie el respeto colectivo hacia las ideas de los demás, con un docente mediador y pluralista que genere impacto social y promueva la transformación social y cultural.

\section{Referencias}

Adoumieh, N. (2015). Modelo discursivo. Programa de escritura a través del currículo. Tesis doctoral inédita en Pedagogía del Discurso. Universidad Pedagógica Experimental Libertador. Instituto Pedagógico de Caracas, Venezuela.

Álvarez Angulo, T. (2005). Didáctica del texto en la formación del profesorado. Madrid: Editorial Síntesis.

Álvarez Angulo, T. \& Ramírez Bravo, R. (2006) Teorías o modelos de producción de textos en la enseñanza y el aprendizaje de la escritura. Didáctica: Lengua y Literatura, 18, 29-60.

Apple, M. (2000). Teoría Crítica y Educación. Buenos Aires: Miño y Dávila Editores.

Bajtín, M. (1979). Estética de la creación verbal. México: Siglo XXI Editores.

Bereiter, C. \& Scardamalia, M. (1992). Two models of classroom learning using a communal database. In S. Dijkstra (Ed.), Instructional models in computer-based learning environments. (pp. 229-241). Berlin: Springer-Verlag.

Bunge, M. (1985). La investigación cientifica. Madrid: Ariel.

Calvo, D. (2006). Modelos teóricos y representación del conocimiento. Memoria para optar al grado de Doctor. Universidad Complutense de Madrid. España.

Carlino, P. (2005). Escribir, leer y aprender en la universidad. Una introducción a la alfabetización académica. Buenos Aires: Fondo de Cultura Económica.

Fairclough, N. (2003). El análisis crítico del discurso como método para la investigación en ciencias sociales. En Wodak, R. y Meyer, M. (ed.). Métodos de análisis critico del discurso. (pp. 179203). Barcelona: Gedisa.

Ferreiro, E. (1994). Diversidad y proceso de alfabetización: De la celebración a la toma de conciencia. Lectura y Vida. Revista Latinoamericana de Lectura, 15(3), 5-11.

Flower, L. \& Hayes, J. (1981). A cognitive process theory of writing. College and communication 32, 365-387.

Foucault, M. (1980). El orden del discurso. España: Cuadernos Marginales.

Giroux, H. (1990). Los profesores como intelectuales. Barcelona: Paidós.

Grice, P. (1975). Lógica y conversación. En Valdés, L. (1991). La búsqueda del significado. (pp. 511 530). Madrid: Tecnos/Universidad de Murcia.

Halliday, M. A. K. (1994). El lenguaje como semiótica social. México: Fondo de Cultura Económica. Jäger, S. (2003). Discurso y conocimiento: Aspectos teóricos y metodológicos de la crítica del discurso y del análisis de dispositivos. En Wodak, R. \& Meyer, M. (ed.). Métodos de análisis critico del discurso. (pp. 61-100). Barcelona: Gedisa. 
Justi, R. (2006). La enseñanza de ciencias basada en la elaboración de modelos. Enseñanza de las ciencias, 24 (2), 174-184. Recuperado de https://bit.ly/2QDecBf.

Link, J. (1983). Was ist und was bringt diskurstaktik. KultuRRevolution, 2, 60-66.

Montolío, E. (2000). Introducción. En: Montolío, E. (Coord). Manual práctico de escritura académica. Vol. II (pp.11-14). Barcelona: Ariel Lingüística.

Morin, E. (1996). Introducción al pensamiento complejo. Barcelona: Gedisa.

Pedroza Flores, R. (2006) La interdisciplinariedad en la universidad. Tiempo de Educar, 7(13), 69-98. Recuperado de https://bit.ly/2RKLbkN

Reyes, G. (1995). El abecé de la pragmática. Madrid: Arco Libros.

Ríos, P. (2004). La Aventura de Aprender. Caracas: Cognitus.

Schön, D. (1992). La formación de profesionales reflexivos. Hacia un nuevo diseño de la enseñanza y el aprendizaje en las profesiones. Barcelona: Paidós.

Stenhouse, L. (1987). Investigación y desarrollo del currículo. Madrid: Visor.

Tolchinsky, L. (1993). Aprendizaje del lenguaje escrito: procesos evolutivos e implicaciones didácticas. Barcelona: Editorial Anthropos.

Tolchinsky, L. \& Simó, R. (2001). Escribir y Leer a través del currículum. Barcelona: Editorial Horsori.

Van Dijk, T. (2003). Ideología y Discurso. Barcelona: Ariel Lingüística.

Vigotsky, L. (1973). Pensamiento y Lenguaje. La Habana: Editorial Pueblo y Educación. 